Supporting Information for manuscript No. ja045506m-3-a-241

\title{
Electric Field Induced Switching Behaviors of Monolayers Modified Silicon Surfaces: Surface Designs and Molecular Dynamics Simulations
}

\author{
Yong Pei, Jing Ma* \\ Department of Chemistry, Institute of Theoretical and Computational Chemistry, Key \\ Lab of Mesoscopic Chemistry of MOE, Nanjing University, Nanjing, 210093, P. R. \\ China
}

I. Parameter sets for the UA models.

II. Figures S1-S4 


\section{Parameter sets for the UA models of $1 C^{\mathrm{im}}-4 C^{\mathrm{im}}$ and $1 F^{\mathrm{im}}-4 F^{\mathrm{im}}$ (c.f. Table 1 ).}

Chain-chain interactions. In the UA approximations, we describe both intra- and inter-molecular interactions among surface-confined chains by a 'united-atom' model in which the $-\mathrm{CH}_{2-},-\mathrm{CF}_{2^{-}}$, and $-\mathrm{COO}^{-}$species are treated as larger pseudoatoms of $\mathrm{CH}_{2}, \mathrm{CF}_{2}$, and $\mathrm{COO}^{-}$with appropriate masses. ${ }^{61}$

The bonded terms including (1) bond stretch, $U_{\text {bond, }}$ (2) angle bend, $U_{\text {bend }}$, and (3) torsion, $U_{\text {torsion }}$, are listed as follows.

$$
U_{\text {bond }}=\frac{k_{b}}{2} \sum_{i=1}^{N-1}\left(\left|\vec{r}_{i+1}-\vec{r}_{i}\right|-d_{0}\right)^{2}
$$

where $d_{0}$ is the natural bond length. The sum run over the $N-1$ bonds and $\vec{r}_{i}$ is the position of the $i$ th site in the chain.

$$
U_{\text {bend }}=\frac{k_{\theta}}{2} \sum_{i=1}^{N-2}\left(\theta_{i+1}-\theta_{0}\right)^{2}
$$

where $\theta_{i+1}$ is the angle formed by bonds connected sites $i, i+1$, and sites $i+1, i+2$. $\theta_{0}$ is the natural angle at site $i+1$.

The torsion term is defined by interactions between four adjacent pseudoatoms, which is represented by the Ryckaert-Bellemans dihedral potential ${ }^{56}$

$$
U_{\text {torsion }}=\sum_{i=1}^{N-3} \sum_{i=0}^{5} C_{i} \cos ^{i}(\varphi)
$$

where $\varphi$ is the dihedral angle between two planes formed by four adjacent sites. There is no site dependence of coefficients $C_{i}$ that the torsion parameters for $\mathrm{X}-\mathrm{CH}_{2}-\mathrm{CH}_{2}-\mathrm{Y}$ are the same regardless of $\mathrm{X}$ and $\mathrm{Y}$ groups. This dihedral potential function has minima at $\varphi=0$ and $\varphi= \pm 2 \pi / 3$, corresponding to the trans and gauche states, respectively. The detailed parameter sets in equations (1)-(3) are shown in Table $\mathbf{S 1 .}$ 
With these values of the $C_{i}$ in Table S1, the energy difference between the trans and gauche states in these hydrogenated chains is $2.9 \mathrm{KJ} / \mathrm{mol}$, with a barrier height of $12.34 \mathrm{KJ} / \mathrm{mol}$. The corresponding trans-gauche energy difference and barrier height existing in the fluorinated chains are estimated to be $8.0 \mathrm{KJ} / \mathrm{mol}$ and $18.0 \mathrm{KJ} / \mathrm{mol}$, respectively. ${ }^{56}$

TableS1. Parameter sets for the bonded terms of the force field

\begin{tabular}{|c|c|c|}
\hline Terms & Atom types & Parameters \\
\hline Bond stretch & $\mathrm{CH}_{2}-\mathrm{CH}_{2}{ }^{\mathrm{a}}$ & $k_{b}=1883.121 \mathrm{KJ} \mathrm{mol}^{-1} \AA^{-2}, d_{0}=1.54 \AA$ \\
\hline \multirow{2}{*}{ Angle bend } & $\mathrm{CH}_{2}-\mathrm{CH}_{2}-\mathrm{CH}_{2}{ }^{\mathrm{b}}$ & $k_{\theta}=460.0 \mathrm{KJ} \mathrm{mol}^{-1} \mathrm{rad}^{-1}, \quad \theta_{0}=111.0^{\circ}$ \\
\hline & $\mathrm{Si}-\mathrm{CH}_{2}-\mathrm{CH}_{2}{ }^{\mathrm{c}}$ & $k_{\theta}=780.0 \mathrm{KJ} \mathrm{mol}^{-1} \mathrm{rad}^{-1}, \quad \theta_{0}=110.0^{\circ}$ \\
\hline \multirow{5}{*}{ Torsion $^{d}$} & $\mathrm{Y} C$ & $C_{0}=9.2789, C_{l}=12.1557, C_{2}=-13.1201$, \\
\hline & $\Lambda-C n_{2}-C n_{2}^{-1}$ & $C_{3}=-3.0597, C_{4}=26.2403, C_{5}=-31.4950$ \\
\hline & $\mathrm{X}-\mathrm{CF}_{2}-\mathrm{CF}_{2}-\mathrm{Y}^{\mathrm{e}}$ & $C_{0}=15.1667, C_{1}=11.9444, C_{2}=-17.3333$, \\
\hline & & $C_{3}=4.4444, C_{4}=34.6667, C_{5}=-48.8889$ \\
\hline & $\mathrm{X}-\mathrm{CH}_{2}-\mathrm{CF}_{2}-\mathrm{Y}^{\mathrm{e}}$ & $C_{0}=11.7644, C_{1}=12.6800, C_{2}=-15.2356$, \\
\hline
\end{tabular}

${ }^{\mathrm{a}}$ The $\mathrm{CH}_{2}-\mathrm{CF}_{2}, \mathrm{CF}_{2}-\mathrm{CF}_{2}, \mathrm{CH}_{2}-\mathrm{COO}^{-}$and $\mathrm{CF}_{2}-\mathrm{COO}^{-}$are assumed to be equivalent in the stretch interaction. This parameter is taken from Ref. 61.

${ }^{\mathrm{b}}$ The bend parameters are also fitted to $\mathrm{CH}_{2}-\mathrm{CF}_{2}-\mathrm{CF}_{2}$ and $\mathrm{CF}_{2}-\mathrm{CF}_{2}-\mathrm{CF}_{2}{ }^{61}$

${ }^{\mathrm{c}}$ The parameters are taken from the universal force field (UFF) ${ }^{62}$

${ }^{d}$ The parameters $C_{0}-C_{5}$ are in unit of $\mathrm{KJ} / \mathrm{mol}$.

${ }^{\mathrm{e}} C_{i}$ in the torsion parameters for $\mathrm{X}-\mathrm{CH}_{2}-\mathrm{CH}_{2}-\mathrm{Y}$ are the same regardless of $\mathrm{X}$ and $\mathrm{Y}$ groups.

The Lennard-Jones (L-J) interaction between sites takes the 12-6 potential function as shown in eq. (4). Values of $\varepsilon$ and $\sigma$ are listed in Table $\mathbf{S 2}$.

$$
U_{L-J}(12-6)=\sum 4 \varepsilon_{i, j}\left[\left(\left(\sigma_{i j} / r_{i j}\right)^{12}-\left(\sigma_{i j} / r_{i j}\right)^{6}\right)\right]
$$

The Coulombic interactions is estimated by 


$$
U_{\text {elec }}=\frac{1}{4 \pi \varepsilon_{0} \varepsilon} \times \frac{q_{i} q_{j}}{\left|\vec{r}_{i}-\vec{r}_{j}\right|}
$$

where $\varepsilon_{0}$ is the permittivity of free space. Charges of pseudoatoms are also listed in Table S2.

Table S2. Non-bonded parameters for pair interactions

\begin{tabular}{|c|c|c|c|}
\hline Atom types & $\varepsilon \quad(K J / \mathrm{mol})$ & $\sigma(n m)$ & $\mathrm{q}(e)$ \\
\hline $\mathrm{CH}_{2}$ & 0.5850 & 0.3905 & \\
\hline $\mathrm{CF}_{2}$ & 0.3370 & 0.5078 & \\
\hline $\mathrm{COO}^{-}$ & 1.229 & 0.4474 & -1.0 \\
\hline $\mathrm{Si}^{\text {a }}$ & 1.730 & 0.4295 & \\
\hline
\end{tabular}

${ }^{\text {a }}$ Parameters are taken from the universal force field (UFF).$^{62}$

${ }^{\mathrm{b}}$ The cross Lennard-Jones parameters for different type of atomic pairs $\left(\mathrm{Si}-\mathrm{CH}_{2}, \mathrm{Si}-\mathrm{COO}^{-}\right.$etc $)$are obtained using the Lorentz-Berthelot (LB) combining rules. ${ }^{53}\left(\varepsilon_{i, j}=\sqrt{\varepsilon_{i} \varepsilon_{j}}, \sigma_{i j}=\frac{\sigma_{i}+\sigma_{j}}{2}\right)$

Monolayer-surface interactions. The silicon surface is represented with four layers of silicon atoms, which is thought to be enough to describe interactions between the silicon surface and monolayer. ${ }^{49-51}$ We neglect the unsaturated dangle bonds on the first layer of the silicon surface. Previous theoretical studies have indicated that the behavior of surface Si atoms has little influence on properties of monolayers. ${ }^{49-51}$ So, we fix all the surface atoms in MD simulations to reduce the calculation costs.

The $-\mathrm{CH}_{2}$ - groups that directly linked to the silicon surface are fixed at positions with the natural bond length of $1.91 \AA$ up to the silicon surface in simulations, which is taken from Universal Force Field (UFF). ${ }^{62}$ Interactions between $\mathrm{Si}$ atoms and surface-confined chains are described by the L-J (12-6) potential with parameters listed in Table S2. The L-J interactions are cut off at the distance of $12 \AA$. 


\section{Figures.}

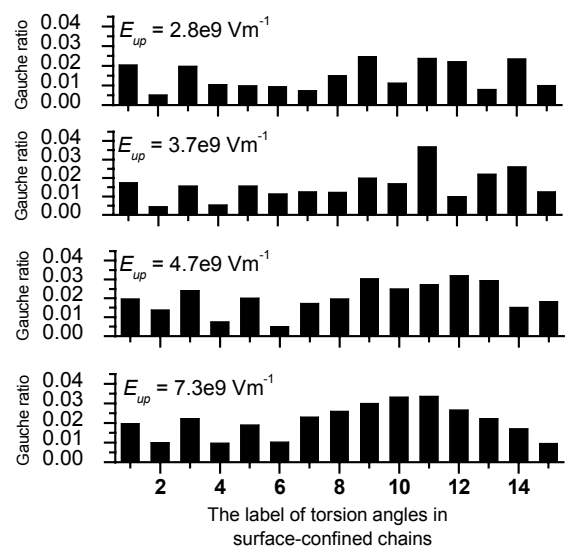

$1 \mathrm{C}$

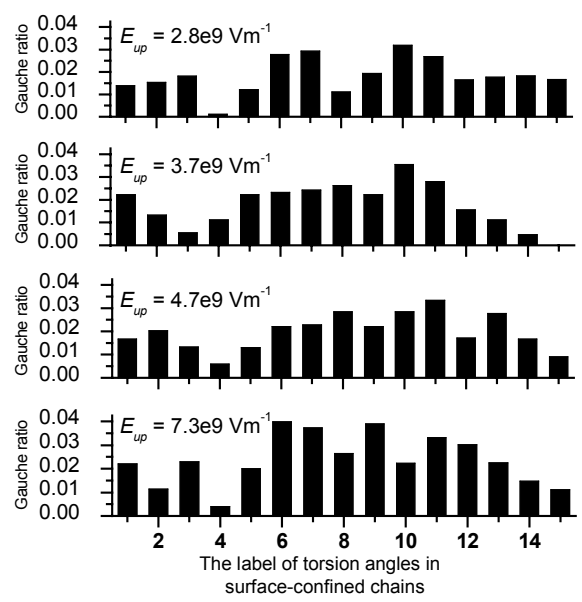

$3 \mathrm{C}$

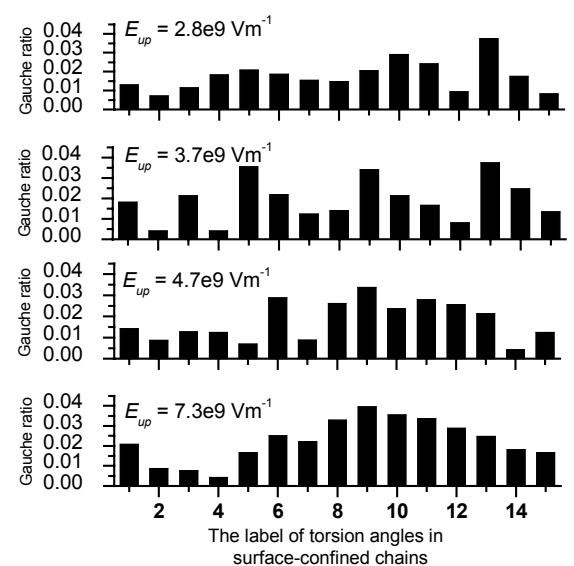

2C

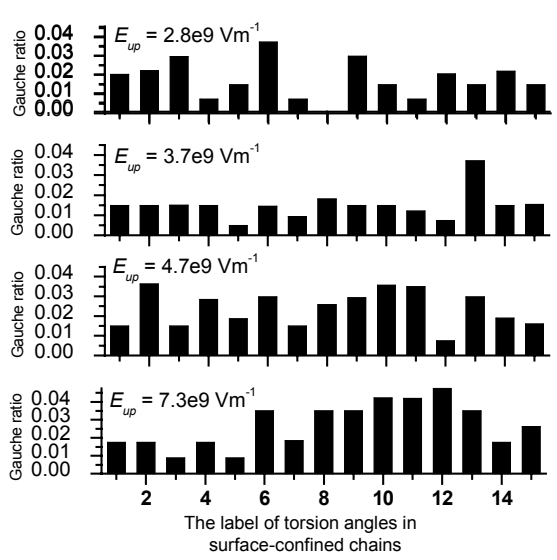

$4 \mathrm{C}$

Figure S1. Distributions of gauche defects in the surface-confined chains when surfaces 1C-4C are restricted in hydrophobic 'off' states. 


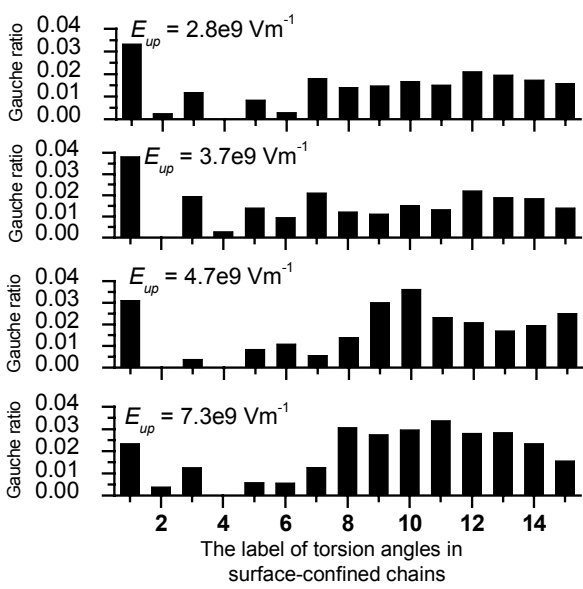

$1 C^{\mathrm{m}}$

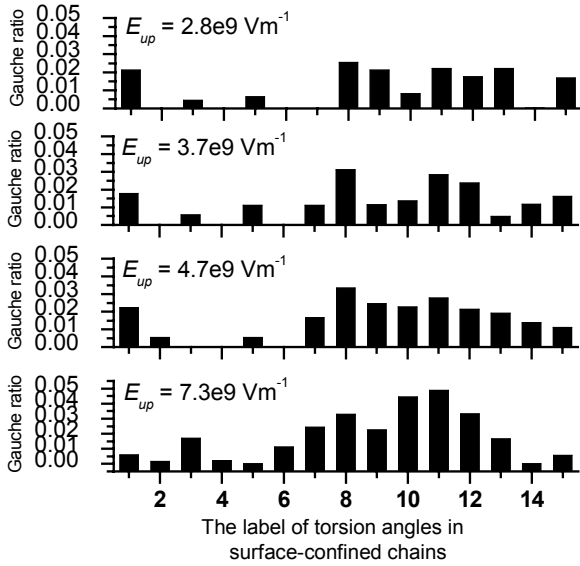

$3 C^{\mathrm{m}}$

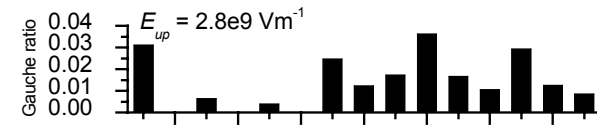

윰 $0.04 \quad \exists E_{u p}=3.7 \mathrm{e} 9 \mathrm{Vm}^{-1}$

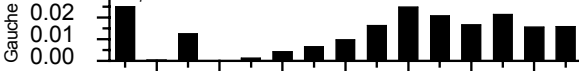

$0.04 \quad \exists E=4.7 \mathrm{e} 9 \mathrm{Vm}^{-1}$

要 0.03

0.01

0.00

윤 0.03

送 0.02

क्ष 0.00

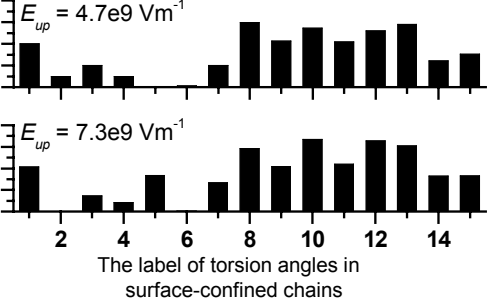

$2 \mathrm{C}^{\mathrm{m}}$

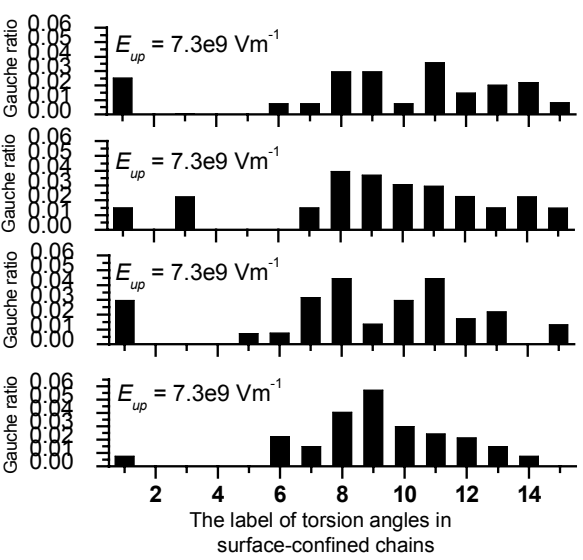

$4 C^{m}$

Figure S2. Distributions of gauche defects in the surface-confined chains when surfaces $\mathbf{1} \mathrm{C}^{\mathrm{m}}-\mathbf{- 4} \mathrm{C}^{\mathrm{m}}$ are restricted in hydrophobic 'off' states. 


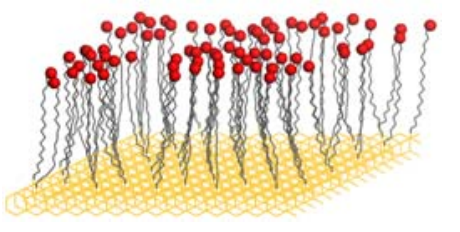

$1 C^{\text {im }}$ (on)

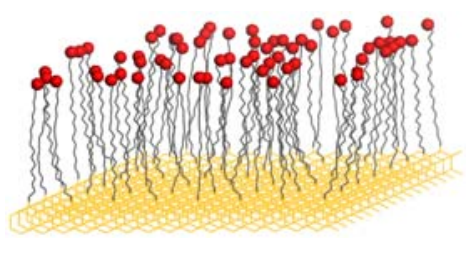

2C im(on)

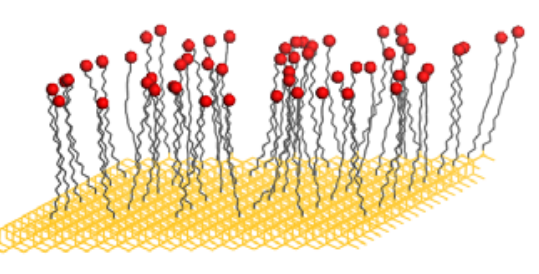

$3 C^{\text {im }}$ (on)

$$
\frac{E_{u p}}{E_{d o w n}}
$$

$$
\underset{E_{\text {down }}}{\stackrel{E_{u p}}{\rightleftharpoons}}
$$

$$
\stackrel{E_{u p}}{\underset{E_{\text {down }}}{\rightleftharpoons}}
$$

$$
\underset{E_{\text {down }}}{\stackrel{E_{u p}}{\rightleftharpoons}}
$$

$$
4 C^{\text {im }} \text { (on) }
$$

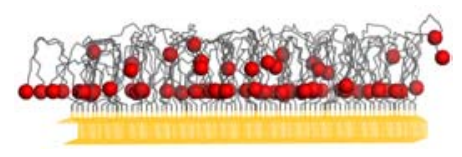

$1 C^{\text {im }}$ (off)

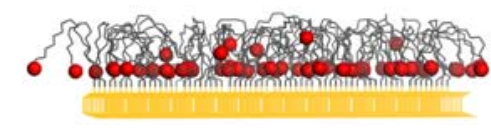

$$
2 C^{\text {im }} \text { (off) }
$$

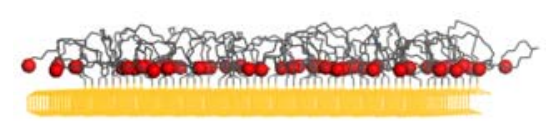

$3 C^{\text {im }}$ (off)

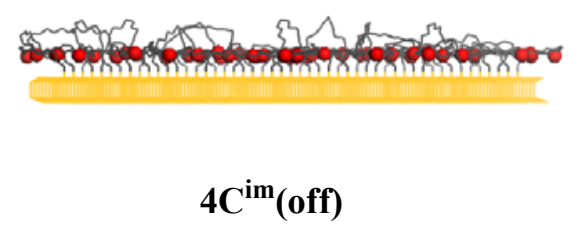

Figure S3. Simulation snapshots of silicon surfaces substituted by single-layer $-\mathrm{CH}_{2}\left(\mathrm{CH}_{2}\right)_{16} \mathrm{COO}^{-}$(c.f. Table $\mathbf{1}, \mathbf{1 C}^{\mathbf{i m}}-\mathbf{4} \mathrm{C}^{\mathrm{im}}$ ) chains in aqueous solution within frameworks of continuum dielectric approximations $(\varepsilon=78)$ and UA models. The $\mathbf{1 C}^{\mathrm{im}}(\mathrm{on})-\mathbf{4} \mathbf{C}^{\mathrm{im}}$ (on) and $\mathbf{1 C ^ { \mathrm { im } }}$ (off)-4C $\mathbf{C}^{\mathrm{im}}$ (off) are equilibrium configurations of surfaces $\mathbf{1 C}^{\mathrm{im}}-\mathbf{4} \mathbf{C}^{\mathrm{im}}$ under $E_{\text {down }}=1.2 \mathrm{e} 9 \mathrm{~V} / \mathrm{m}$ and $E_{u p}=3.7 \mathrm{e} 9 \mathrm{~V} / \mathrm{m}$, respectively. The red CPK groups represent the $-\mathrm{COO}^{-}$groups. 


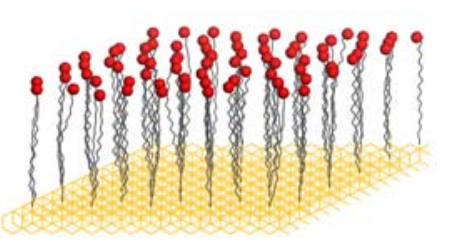

$1 F^{\text {im }}$ (on)

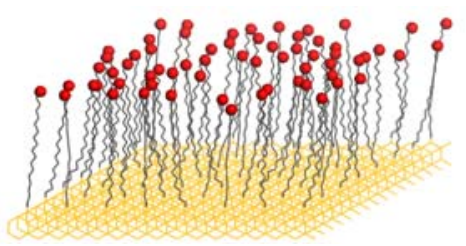

$2 F^{\text {im }}$ (on)

$$
\underset{E_{\text {down }}}{\stackrel{E_{u p}}{\rightleftharpoons}}
$$

$\underset{E_{\text {down }}}{\stackrel{E_{u p}}{\rightleftharpoons}}$

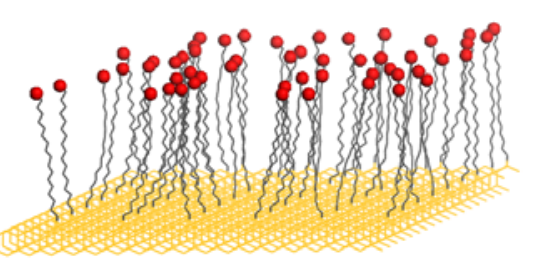

$3 F^{i m}$ (on)

$$
\underset{E_{\text {down }}}{\stackrel{E_{u p}}{\rightleftharpoons}}
$$

$4 F^{\text {im }}$ (on)

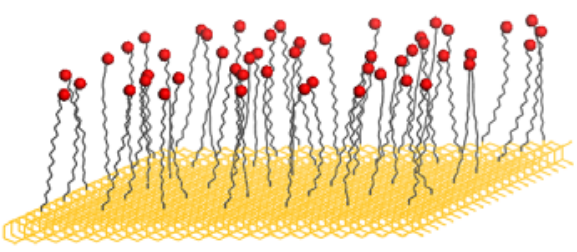

4F (on)

$$
2 F^{i m}(o f f)
$$

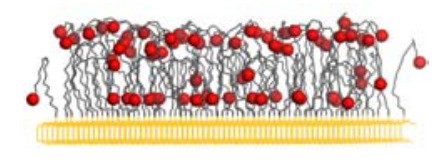

$$
1 F^{\text {im }} \text { (off) }
$$
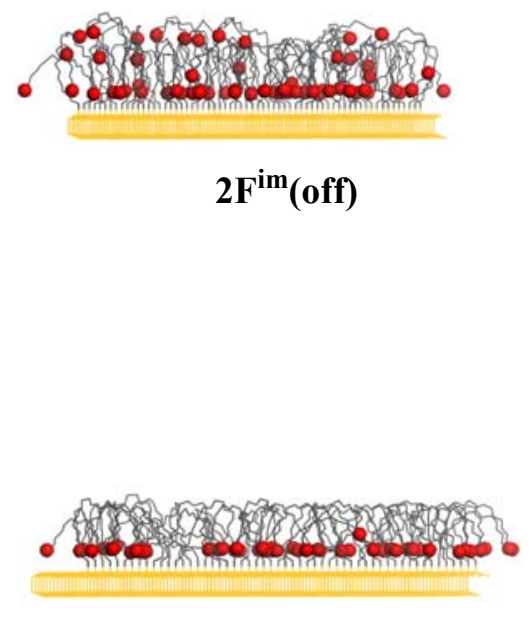

$3 F^{\text {im }}($ off $)$$$
\underset{E_{\text {down }}}{\stackrel{E_{u p}}{\rightleftharpoons}}
$$
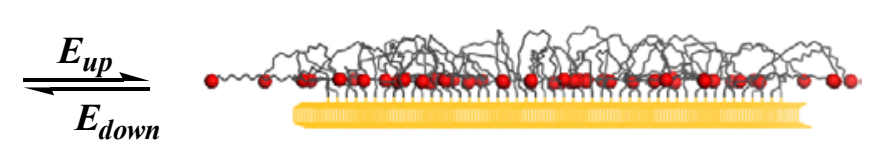

$4 F^{\text {im }}$ (off)

Figure S4. Simulation snapshots of silicon surfaces substituted by single-layer

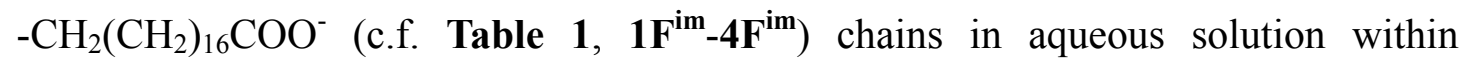
frameworks of continuum dielectric approximations $(\varepsilon=78)$ and UA models. The $\mathbf{1 F}$ (on)-4F $\mathbf{F}^{\mathrm{im}}$ (on) and $\mathbf{1 F ^ { \mathrm { im } }}$ (off)-4F $\mathbf{F}^{\mathrm{im}}$ (off) are equilibrium configurations of surfaces $\mathbf{1 F}^{\mathrm{im}}-\mathbf{4} \mathbf{F}^{\mathrm{im}}$ under $E_{\text {down }}=1.2 \mathrm{e} 9 \mathrm{~V} / \mathrm{m}$ and $E_{u p}=3.7 \mathrm{e} 9 \mathrm{~V} / \mathrm{m}$, respectively. The red CPK groups represent the $-\mathrm{COO}^{-}$groups. 